\title{
GW23-e1610 FOLIC ACID REDUCES ADHESION MOLECULES VCAM-1 EXPRESSION ON ENDOTHELIUM IN RATS WITH HYPERHOMOCYSTEINEMIA
}

doi:10.1136/heartjnl-2012-302920a.141

${ }^{1}$ Wang Yuqin, ${ }^{2}$ Li Ming. ${ }^{1}$ The PLA252 Hospital Department of Cardiology, BaoDing 071000; 'Institute of Cardiology, Union Hospital, Tongji Medical College, Huazhong University of Science and Technology, Wuhan

Objectives To investigate the effects of folic acid supplementation on the expression of adhesion molecules VCAM 1 in aortic endothelium of rats with hyperhomocys teinemia (HHCY) induced by ingestion of excess methionine (MET).

Methods Thirty male SD rats $(200 \pm 20 \mathrm{~g})$ were divided into 3 groups ( $n=10$ in each group): control group (Control), high MET group (MET) and MET plus folate group (MET + folate). The rats were fed respectively on normal diet, normal diet enriched by $17 \mathrm{~g} / \mathrm{kg}$ MET and normal diet plus $17 \mathrm{~g} / \mathrm{kg}$ MET and $0.06 \mathrm{~g} / \mathrm{kg}$ folate for $45 \mathrm{~d}$. Thelevels of total plasma homocysteine (HCY) were detected and the expression of VCAM-1 protein and mRNA in aorta of rats was detected respectively by immunohistochemistry and RT - PCR.

Results The high-methionine diet resulted in a significant increase in the plasma HCY levels $((140.7 \pm 36.9)$ iamol/1 vs $(6.5 \pm 1.1)$ iamol/1). The serum HCY levels were significantly lower in rats fed on high-methionine plus folate-rich diet than those in rats fed on the high-methionine diet $(50.6 \pm 21.8) \mathrm{iamol} / \mathrm{l}$ vs $(140.7 \pm 36.9)$ iamol/1, $p<0.05$. The expression of adhesion molecules VCAM-1 at protein and mRNA levels was higher in aortic endothelium of rats fed on the high-methionine diet than that in control rats. The expression ofVCAM-1 at protein and mRNA levels was significantly reduced in aortic endothelium of rats fed on high-methionine plus folate-rich diet compared with that in rats fed on high methionine diet.

Conclusions A high methionine diet for 45 dissufficient to induce HHCY. Folate supplementation to the rats fed on the high-methenine diet prevents the elevation of HCY levels in the blood and reduces the expression of adhesion molecules VCAM- 1 in aorta of rats with HHCY. 